\title{
Aplicação de Algoritmo de Busca Cuco no Dimensionamento Ótimo de Gerador Fotovoltaico para Redução de Custos
}

\author{
Mariana R. B. Alencar. Benemar A. Souza. Washington L. A. Neves, Rafael S. F. Ferraz \\ Programa de Pós-Graduação em Engenharia Elétrica, Universidade Federal de Campina Grande \\ Campina Grande, PB (email: mariana.alencar@ee.ufcg.edu.br)
}

\begin{abstract}
Faced with the growing demand for electricity, renewable energy sources are an increasingly attractive option. The optimal sizing of distributed generation problem can be considered through different objectives, but in this work the objective function is the total costs. Therefore, a Cuckoo Search Algorithm is used to determine the optimal size (model and number of modules) of a photovoltaic generator to be installed in a hotel so the total annual costs are minimal. For this, real data of solar irradiance, ambient temperature and demand are used by Monte Carlo simulation to consider the randomness of these variables. The economic viability of the project is determined by the calculation of the levelized cost of electricity and the payback time.
\end{abstract}

Resumo: Diante da crescente demanda por energia elétrica, as fontes renováveis de energia são uma opção cada vez mais atrativa. O problema do dimensionamento ótimo de geração distribuída pode ser considerado por meio de diferentes objetivos, porém neste trabalho a função objetivo são os custos totais. Sendo assim, um algoritmo de busca Cuco é empregado para determinar o dimensionamento ótimo de um gerador fotovoltaico (modelo e quantidade de módulos) a ser instalado em um hotel a fim de que os custos totais anuais sejam mínimos. Para tanto, dados reais de irradiância solar, temperatura ambiente e demanda são utilizados mediante simulação de Monte Carlo para considerar a aleatoriedade dessas variáveis. A viabilidade econômica do projeto é determinada mediante o cálculo do custo nivelado de energia ou LCOE (do inglês Levelized Cost of Electricity) e do tempo de retorno do investimento.

Keywords: Cuckoo Search; Monte Carlo simulation; photovoltaic generation; optimal sizing; costs reduction.

Palavras-chaves: Busca Cuco; simulação de Monte Carlo; geração fotovoltaica; dimensionamento ótimo; redução de custos.

\section{INTRODUÇÃO}

O papel que a energia desempenha em quase todas as atividades humanas é amplamente conhecido. Sem uma fonte de energia confiável e acessível, governos, instituições e indivíduos teriam dificuldades em realizar suas atividades sociais e econômicas. O custo de energia é um componente vital de um empreendimento comercial. Com práticas e tecnologias de eficiência energética, o custo da energia pode ser reduzido sem necessariamente reduzir o conforto dos usuários (Oluseyi et al. 2016).

O setor hoteleiro no Brasil é um segmento importante da economia. Em 2016, havia 31,3 mil estabelecimentos de hospedagem no país, com 1 milhão de unidades habitacionais (suítes, quartos e chalés) e 2,4 milhões de leitos (IBGE, 2016). Dentre as edificações comerciais ou de serviços, o setor hoteleiro representa uma quantidade bastante significativa de consumo de energia (Stoppato et al. 2016).

Para que a sazonalidade do fluxo de turistas não cause transtornos às cidades e aos seus habitantes é importante um planejamento energético, preferencialmente com a utilização de energia limpa e renovável, nos meios de hospedagem. Além disso, tal planejamento representa avanço expressivo em termos de posicionamento e visibilidade dentro do mercado turístico, gerando resultados financeiros positivos (Nishimoto e Varajão, 2018).

Chow et al. (2003) descrevem um estudo comparativo de três opções distintas de aplicação de tecnologia fotovoltaica (FV) integrada em grande escala em uma cidade costeira no mar da China meridional. O modelo computacional foi baseado em uma parede fotovoltaica de silício monocristalino de $260 \mathrm{~m}^{2}$ em um edifício de 30 andares e foi aplicado a um hotel hipotético da cidade de Macau, China. A análise númerica foi realizada por meio de software específico. Os resultados apontam que as diferentes opções de projeto exibem diferenças de desempenho elétrico a curto prazo, mas têm rendimentos similares a longo prazo.

Dalton et al. (2009) apresentam uma análise de viabilidade técnico-financeira de configurações de alimentação diversas para um hotel de grande porte conectado: i) somente pela rede de distribuição; ii) somente solar e eólica e iii) híbrida (eólica, solar e rede de distribuição). Os critérios de avaliação incluíram o custo presente líquido e o tempo de retorno. O software HOMER $^{\circledR}$ foi utilizado como ferramenta de avaliação, com modelagem de dados de carga horária de um hotel localizado em uma área subtropical de Queensland, 
Austrália. Os resultados demonstraram que a geração eólicasolar têm potencial de fornecer parte significativa da energia consumida em uma instalação turística de grande porte, embora não dispense a interligação à rede de distribuição.

A revisão da literatura fornece poucos trabalhos que utilizam métodos de otimização para realizar apenas o dimensionamento ótimo de geração fotovoltaica com a intenção de reduzir os custos totais anuais. Até o momento os autores tem conhecimento dos trabalhos de Qi et al. (2008), Liu et al. (2011), Stoppato et al. (2016), Oliveira e Maria (2017), que utilizam como métodos de otimização o algoritmo de evolução diferencial (ED), algoritmo genético (AG), otimização por enxame de partícula (PSO) e sistema imunológico artificial (SIA), respectivamente.

Como o número de parâmetros a serem ajustados pelo algoritmo de Busca Cuco (BC) é menor do que AG e PSO, é mais genérico e tem potencial para se adaptar a uma gama de problemas de otimização. Portanto, o algoritmo BC foi escolhido para resolver o problema do dimensionamento ótimo de um gerador fotovoltaico, considerando as incertezas de carga e insumo de geração por meio da simulação de Monte Carlo.

O trabalho está organizado assim: na seção 2 o problema é enunciado; a seção 3 é uma breve explanação sobre o algoritmo de otimização utilizado; o algoritmo de solução completo é apresentado na seção 4 e aplicado na seção 5; a seção 6 é a conclusão.

\section{ENUNCIADO DO PROBLEMA}

Ao se pensar em investir em um projeto, existe a necessidade de verificar sua viabilidade econômica. Os custos com energia elétrica geralmente são elevados em hotéis devido a vários fatores tais como: necessidade de aquecimento ou resfriamento dos ambientes, aquecimento de água, área de lazer e restaurantes. Portanto, medidas que diminuam tais gastos devem ser analisadas e posteriormente aplicadas.

O problema tratado aqui pode ser enunciado do seguinte modo: determinar o melhor modelo e a quantidade ideal de módulos fotovoltaicos a serem instalados em uma determinada área, de modo que os custos totais com energia elétrica sejam mínimos. Desse modo, o problema de otimização consiste em:

$$
\begin{aligned}
\operatorname{Minimizar} C_{T} & =C_{I}+C_{M}+C_{E C R}-C_{E P} \\
C_{I} & =C_{\text {mod }}+C_{d i v}
\end{aligned}
$$

sujeito a:

$$
\begin{aligned}
A_{G} & <1000 \mathrm{~m}^{2} \\
P_{\text {inst }} & <0,3 \cdot P_{\text {pico }}
\end{aligned}
$$

sendo:

$C_{T}: \quad$ custos totais;

$C_{I}: \quad$ custo de instalação do sistema FV;

$C_{M}: \quad$ custo de manutenção do sistema FV igual a $1 \%$ de $C_{I}$;

$C_{E C R}: \quad$ custo de energia comprada da concessionária;

$C_{E P}: \quad$ custo da energia produzida pelo sistema $\mathrm{FV}$;
$C_{\text {mod }}: \quad$ custo dos módulos fotovoltaicos;

$C_{d i v}$ : custos diversos (inversores, estruturas metálicas e suporte, custos e despesas administrativas e outros componentes);

$A_{G}: \quad$ área total ocupada pelo gerador $\left(\mathrm{m}^{2}\right)$;

$P_{\text {inst }}$ : potência nominal instalada do gerador fotovoltaico

(W);

$P_{\text {pico }}$ : potência de pico do empreendimento (W);

Dentre as possíveis restrições do problema, se considera a restrição de área disponível para a instalação dos módulos e a máxima penetração de geração distribuída (GD). Para efeito de evitar sobrecarga do sistema de distribuição por elevada penetração fotovoltaica, baseado em Atwa et al. (2010), o limite de potência instalada de GD é $30 \%$ da carga de pico. Desconsidera-se a remuneração do capital próprio, ou o custo do financiamento, necessário para a instalação do sistema FV e considera-se que os custos de instalação serão pagos ao longo do ano analisado.

O cálculo da energia gerada e demandada é realizado por meio da simulação de Monte Carlo, considerando o período de análise de um ano.

\subsection{Dados de Carga}

Os dados considerados dizem respeito ao consumo de energia elétrica de um hotel disponível em Shan (2019) que correspondem a medições horárias ao longo de um ano. Considera-se que a carga segue uma distribuição de probabilidade normal. A partir dos dados de medição é possível determinar o valor médio e o desvio-padrão da amostra. O hotel está situado hipoteticamente no mesmo local ou bem próximo em que foram feitas as medições de irradiância solar e temperatura ambiente.

\subsection{Modelo de Geração}

A potência de saída do módulo fotovoltaico depende da irradiância solar e temperatura ambiente, bem como das características do próprio módulo. Sendo assim, a potência de saída é calculada por (Atwa et al. 2010):

$$
\begin{gathered}
P(s)=N F_{P} V I \\
F_{P}=\frac{V_{M P P T} I_{M P P T}}{V_{o c} I_{s c}} \\
V=V_{o c}-K_{v} t_{c} \\
I=s \cdot\left[I_{s c}+K_{i}\left(t_{c}-25\right)\right] \\
t_{c}=t_{A}+s \cdot\left(\frac{N_{O T}-20}{0,8}\right)
\end{gathered}
$$

em que:

$\begin{array}{ll}t_{c}: & \text { temperatura do módulo } \mathrm{FV}\left({ }^{\circ} \mathrm{C}\right) ; \\ t_{A}: & \text { temperatura ambiente }\left({ }^{\circ} \mathrm{C}\right) ; \\ s: & \text { irradiância solar }\left(\mathrm{W} / \mathrm{m}^{2}\right) ;\end{array}$


$N_{O T}: \quad$ temperatura nominal de operação $\left({ }^{\circ} \mathrm{C}\right)$;

$I_{s c}: \quad$ corrente de curto circuito (A);

$V_{o c}: \quad$ tensão de circuito aberto $(\mathrm{V})$;

$K_{i}: \quad$ coeficiente de temperatura da corrente $\left(\mathrm{A} /{ }^{\circ} \mathrm{C}\right)$;

$K_{v}: \quad$ coeficiente de temperatura da tensão $\left(\mathrm{V} /{ }^{\circ} \mathrm{C}\right)$;

$F_{P}$ : fator de preenchimento, que é um parâmetro de avaliação de desempenho da célula e depende do material e da técnica de fabricação;

$V_{M P P T}$ : tensão no ponto de máxima potência $(\mathrm{V})$;

$I_{M P P T}$ : corrente no ponto de máxima potência (A);

$N$ : $\quad$ número de módulos utilizado no sistema;

$P(s)$ : potência de saída do módulo $\mathrm{FV}(\mathrm{W})$;

A potência gerada, conforme (5), é função das variáveis aleatórias irradiância solar e temperatura ambiente, para as quais se consideram funções de distribuição de probabilidade normal distintas.

\section{ALGORITMO DE BUSCA CUCO}

O algoritmo de Busca Cuco é um algoritmo de otimização inspirado no comportamento reprodutivo de algumas espécies de cuco, introduzido por Yang e Deb (2009). As fêmeas de cuco depositam seus ovos em ninhos de outras aves, que podem ou não ser da mesma espécie. Se o pássaro hospedeiro descobre o ovo no ninho, pode destruí-lo ou abandonar o ninho e construí-lo em outro local.

Para reduzir a probabilidade de descobrimento do ovo pelo pássaro hospedeiro, os cucos usam várias estratégias como imitar as cores dos ovos do hospedeiro, escolher os melhores ninhos e desenvolver a habilidade de imitar o canto do hospedeiro para ganhar mais oportunidade de alimentação (Nadjemi et. al. 2017). A principal analogia presente nesta metaheuristica é a identificação dos ninhos como possíveis soluções para o problema de otimização. O parasitismo do cuco pode ser resumido nas três regras abaixo (Yang e Deb, 2009):

- Cada cuco deposita um ovo a cada vez e o coloca em um ninho escolhido aleatoriamente;

- Os melhores ninhos com ovos de alta qualidade serão passados para as próximas gerações;

- O número de ninhos hospedeiros disponíveis é fixo, e o ovo depositado pelo cuco é descoberto pelo hospedeiro com uma probabilidade de descobrimento $\mathrm{p}_{\mathrm{a}} \in[0,1]$.

Uma nova solução é criada a partir de uma solução existente e do voo de Lévy:

$$
x_{i}^{t+1}=x_{i}^{t}+\alpha \oplus \operatorname{Lé} v y(\lambda)
$$

em que $\alpha$ é o tamanho do passo que controla a escala do voo e $\bigoplus$ indica uma multiplicação termo a termo das entradas. A equação (10) é essencialmente uma cadeia de Markov, uma vez que a próxima localização depende somente da localização atual e de uma probabilidade de transição, dado pelo primeiro e segundo termos de (10), respectivamente.

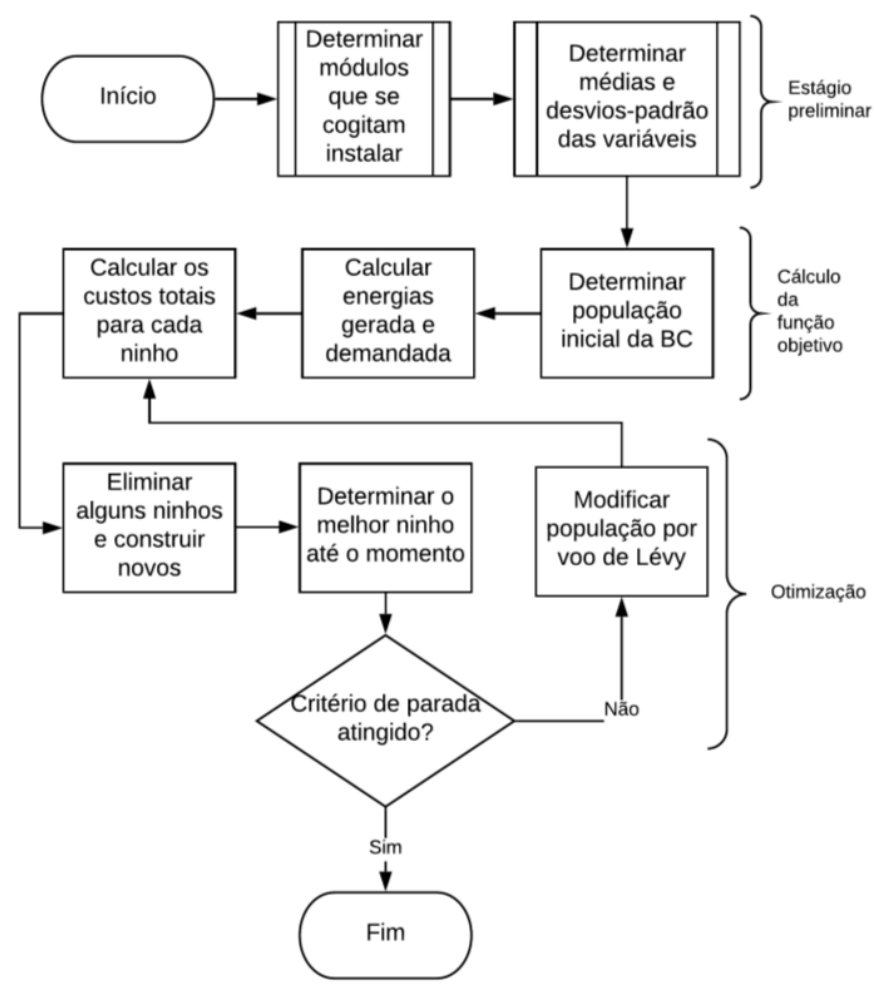

Fig. 1 Fluxograma do algoritmo de solução

A busca global do algoritmo é realizada mediante voos de Lévy, que são passos aleatórios cujo tamanho é obtido por meio de uma distribuição de Lévy:

$$
\operatorname{Lé} v y(\lambda) \sim u=t^{-\lambda}, \quad(1<\lambda \leq 3)
$$

Algumas das novas soluções devem ser geradas por voo de Lévy nas proximidades da melhor solução encontrada até o momento, o que acelera a busca local. No entanto, uma fração considerável das novas soluções devem ser geradas longe da melhor solução atual, para que o algoritmo não fique preso em um mínimo local (Yang e Deb, 2009).

\section{ALGORITMO DE SOLUÇÃO}

Apesar de ter uma base de dados anual tanto de consumo de energia quanto de irradiância e temperatura, é necessário considerar a aleatoriedade das variáveis. Para tanto, toma-se a média e o desvio-padrão das amostras e números pseudoaleatórios com distribuição normal são gerados. A cada simulação de Monte Carlo se tem novos valores de demanda, irradiância solar e temperatura ambiente. O cálculo da energia gerada e demandada deve ser refeito sempre que uma possível solução for determinada pelo algoritmo de otimização. $\mathrm{O}$ algoritmo proposto é o seguinte:

1. Determinar os módulos que se cogitam instalar: modelos, dados e preços;

2. A partir das bases de dados disponíveis, determinar médias e desvios-padrão das variáveis irradiância solar, temperatura ambiente e demanda;

3. Determinar aleatoriamente a população inicial do algoritmo $\mathrm{BC}$; 
4. Calcular a energia gerada pelo sistema FV e demandada pelo empreendimento por meio da simulação de Monte Carlo, considerando o período de um ano;

5. Calcular a função objetivo $C_{T}$ empregando (1);

6. Determinar o melhor ninho até o momento, eliminar alguns ninhos a partir da probabilidade de descobrimento e construir novos ninhos empregando voo de Lévy;

7. Repetir os passos de 4 a 6 até que o critério de parada seja atendido.

Os pontos 1 a 3 são estágios preliminares que são requeridos apenas na primeira vez que a função objetivo é calculada. Os passos de 4 a 7 são executados durante todo o processo. A Fig. 1 é o fluxograma do algoritmo de solução proposto.

\section{APLICAÇÃO DO MÉTODO}

\subsection{Dados e Hipóteses}

A partir de uma realização de pesquisa de mercado, oito modelos de módulos fotovoltaicos foram selecionados como candidatos à instalação. Obviamente, mais modelos poderiam ter sido selecionados, inclusive com outras potências nominais. Os dados dos módulos são mostrados na tabela 1 , em que os preços estão arredondados.

Tabela 1. Dados dos módulos considerados

\begin{tabular}{lcccccccc}
\hline & \multicolumn{7}{c}{ Modelos } \\
\cline { 2 - 9 } & 1 & 2 & 3 & 4 & 5 & 6 & 7 & 8 \\
\hline \hline$P_{\text {nom }}(\mathrm{W})$ & 275 & 325 & 270 & 280 & 330 & 270 & 260 & 395 \\
\hline$V_{O C}(\mathrm{~V})$ & 37,9 & 45,5 & 37,9 & 38,2 & 46,1 & 39 & 37,65 & 47 \\
\hline$I_{S C}(\mathrm{~A})$ & 9,32 & 9,34 & 9,27 & 9,45 & 9,38 & 9,13 & 9,11 & 10,82 \\
\hline$N_{O T}\left({ }^{\circ} \mathrm{C}\right)$ & 45 & 45 & 45 & 46 & 45 & 45 & 45 & 42 \\
\hline$K_{v}\left({ }^{\circ} \mathrm{C}\right)$ & 117,8 & 141 & 121,3 & 122,4 & 147,5 & 120,9 & 128 & 136,3 \\
\hline$K_{i}\left({ }^{\circ} \mathrm{C}\right)$ & 4,7 & 5 & 4,6 & 4,7 & 4,7 & 6,4 & 5,9 & 5,4 \\
\hline$F_{P}$ & 0,78 & 0,76 & 0,77 & 0,78 & 0,76 & 0,76 & 0,76 & 0,78 \\
\hline $\begin{array}{l}\text { Preço } \\
(\mathrm{R} \$)\end{array}$ & 492 & 582 & 483 & 501 & 591 & 483 & 465 & 707 \\
\hline Para & & & & & & & & \\
\hline
\end{tabular}

Para considerar os custos diversos (inversores, estruturas de suporte, dentre outros) buscou-se na literatura uma estimativa de tais valores. O relatório técnico divulgado pelo Instituto Ideal e AHK-RJ (2018) é uma pesquisa com 359 empresas brasileiras instaladoras, projetistas ou revendedoras de componentes da geração fotovoltaica.

A partir dos dados coletados, foi possível afirmar que o preço médio do Wp para sistemas com potência instalada entre $31 \mathrm{e}$ $100 \mathrm{~kW}$ é R \$ 4,72 por Wp. Deste valor, $28 \%$ corresponde ao custo com os módulos, $21 \%$ corresponde aos inversores, $14 \%$ ao projeto e a instalação, $10 \%$ estruturas metálicas e suporte, $10 \%$ aos custos e despesas administrativas, e $7 \%$ corresponde a outros custos. Os dados acima são de 2017 e apesar de poderem ter sofrido alterações, foram empregados no presente trabalho por serem os mais recentes encontrados na literatura brasileira.

\subsection{Detalhes do Algoritmo de Solução}

Com relação ao algoritmo de Busca Cuco foram adotados:

- Ninhos com representação decimal, em que uma solução possível é a da fig. 2: instalar 230 módulos do modelo 2;

- Número de ninhos igual a 25;

- $\quad$ Probabilidade $p_{a}$ igual a $35 \%$;

- Número total de iterações (critério de parada) igual a 250.

\begin{tabular}{|c|l|}
\hline 2 & \multicolumn{1}{|c|}{230} \\
\hline modelo escolhido & $\begin{array}{l}\text { quantidade de } \\
\text { módulos a ser } \\
\text { instalados }\end{array}$ \\
\hline
\end{tabular}

Fig. 2 Solução possível para o problema de dimensionamento

As simulações de Monte Carlo foram em número igual ao total de horas do ano: 8760 . Os parâmetros média $(\mu)$ e desviopadrão $(\sigma)$ das variáveis aleatórias estão mostrados na tabela 2 e foram determinados mediante bases de dados anuais.

Diante do exposto na seção 5.1, os custos diversos foram estimados em R\$ 2,60 por Wp e a tarifa cobrada pela concessionária é considerada a tarifa local para empreendimentos comerciais no valor de $\mathrm{R} \$ / \mathrm{kWh} 0,5074 \mathrm{e}$ que esta não se altera ao longo do ano simulado.

Tabela 2. Parâmetros das variáveis aleatórias

\begin{tabular}{cccc}
\hline Parâmetro & $\begin{array}{c}\text { Irradiância } \\
\text { solar }\left(\mathrm{W} / \mathrm{m}^{2}\right)\end{array}$ & $\begin{array}{c}\text { Temperatura } \\
\text { ambiente }\left({ }^{\circ} \mathrm{C}\right)\end{array}$ & $\begin{array}{c}\text { Demanda } \\
\text { energética } \\
(\mathrm{kWh} / \mathrm{ano})\end{array}$ \\
\hline \hline$\mu$ & 441 & 23,63 & 611 \\
\hline$\sigma$ & 287,9 & 3,23 & 219 \\
\hline
\end{tabular}

\subsection{Resultados}

Como se trata de uma abordagem probabilística, tanto a energia gerada quanto a demandada são variáveis ao longo do ano. Sendo assim, o custo anual com energia elétrica é contabilizado a partir da diferença entre a energia gerada durante o ano e a energia demandada no mesmo período. Os custos anuais com energia elétrica sem a geração fotovoltaica, se considerarmos o consumo medido disponível na base de dados, seriam iguais a $\mathrm{R} \$ 2,72$ milhões.

Para analisar a evolução do algoritmo de otimização, o número total de iterações foi subdividido em dez épocas, semelhante àquelas empregadas em AG. Os resultados da melhor simulação são mostrados na tabela 3 , em que uma solução se repetiu da primeira a sexta época e a solução ótima se repetiu da sétima à décima época.

A tabela 4 é um resumo da execução do método por dez vezes. Assim é possível analisar o valor médio da função objetivo e seu desvio-padrão. 
Tabela 3. Resultado da simulação

\begin{tabular}{cccc}
\hline Época & $\begin{array}{c}\text { Modelo do } \\
\text { módulo }\end{array}$ & $\begin{array}{c}\text { Quantidade } \\
\text { de } \\
\text { módulos }\end{array}$ & $\begin{array}{c}\text { Custos } \\
\text { anuais } \\
\text { totais }(\mathrm{R} \$ \\
\left.10^{6}\right)\end{array}$ \\
\hline \hline Inicial & 7 & 305 & 2,94 \\
\hline $1-6$ & 3 & 247 & 2,87 \\
\hline $7-10$ & 3 & 250 & 2,86 \\
\hline
\end{tabular}

Tabela 4. Resumo do resultado das simulações

\begin{tabular}{ccccc}
\hline $\begin{array}{c}\text { Função } \\
\text { objetivo } \\
\left(\mathrm{R} \$ 10^{6}\right)\end{array}$ & Média & $\begin{array}{c}\text { Pior } \\
\text { solução }\end{array}$ & $\begin{array}{c}\text { Melhor } \\
\text { solução }\end{array}$ & $\begin{array}{c}\text { Desvio- } \\
\text { padrão }\end{array}$ \\
\hline$C_{T}$ & 2,88 & 3,00 & 2,86 & 0,0196 \\
\hline
\end{tabular}

\subsection{Análise da Viabilidade Econômica}

Os custos anuais expostos na tabela 3 se referem ao primeiro ano de implantação do sistema FV e incluem todos os custos, como expresso em (1). Para avaliar a viabilidade econômica da implementação é importante calcular o custo nivelado de energia (LCOE) e o payback (tempo de retorno do investimento). Para tanto, utiliza-se a solução ótima que é instalar 250 módulos do modelo 3.

O custo da energia elétrica produzida durante o tempo de uma unidade produtora, seja ela de origem renovável ou não, designidado por custo nivelado de energia (LCOE), é o principal indicador que permite comparar tecnologias de produção (Pereira et al. 2016). Por meio do LCOE se calcula os custos totais de um sistema produtor de energia durante o tempo de vida do sistema, incluindo os custos de investimento, manutenção e operação e compara esse custo com a estimativa de eletricidade que o sistema irá produzir durante o mesmo período.

O LCOE pode ser calculado por:

$$
L C O E=\frac{C_{0}+\sum_{t=1}^{n} \frac{C_{t}(1+i)^{t}}{(1+j)^{t}}}{\sum_{t=1}^{n} \frac{E_{t}(1+d)^{t}}{(1+j)^{t}}}
$$

sendo:

$t$ : $\quad$ período considerado, onde n é o tempo máximo de vida útil do sistema;

$C_{0}$ : $\quad$ custo inicial de instalação $(\mathrm{R} \$)$;

$C_{t}: \quad$ custo do sistema FV no período $t$;

$i$ : inflação anual considerada;

$E_{t}$ : energia gerada pelo empreendimento no período $t$;

$d: \quad$ degradação do sistema FV;

$j: \quad$ taxa de desconto.

O custo de instalação é de R\$296,33 • $10^{3}$, o período considerado é de 25 anos, a inflação considerada é de $3,75 \%$ a.a, a degradação de $0,7 \%$ a.a, a taxa de desconto de $6,5 \%$ a.a que corresponde à taxa Selic do ano de 2018.
Para tal situação, o custo nivelado de energia é R\$ $0,1153 / \mathrm{kWh}$. Se compararmos com o custo da energia convencional, que é de $\mathrm{R} \$ 0,5074 / \mathrm{kWh}$, tem-se uma redução de $77,28 \%$ no custo da energia.

Outro indicador de viabilidade econômica é o tempo de retorno do investimento (payback time) e é simplesmente o tempo necessário para que um projeto zere o fluxo de caixa, ou seja, o tempo em que o somatório de todas as receitas se igualem aos custos (Veríssimo, 2017).

O payback descontado considera o valor monetário no tempo pois utiliza uma taxa de desconto para verificar o período exato em que o projeto recupera o valor inicial investido. A tabela 5 é o fluxo de caixa projetado, em que a economia na fatura de energia considera a taxa de degradação de $0,7 \%$ e a taxa de desconto de 6,5\%. O payback descontado do projeto é de 2 anos e 10 meses, um tempo relativamente baixo, como era esperado, haja vista os custos de instalação de sistemas FV reduziram bastante ao longo dos anos.

Tabela 5. Fluxo de caixa projetado

\begin{tabular}{ccccc}
\hline Ano & Mês & $\begin{array}{c}\text { Economia na } \\
\text { fatura de } \\
\text { energia }(\mathrm{R} \$ \\
\left.10^{3}\right)\end{array}$ & $\begin{array}{c}\text { Fluxo de } \\
\text { caixa }(\mathrm{R} \$ \\
\left.10^{3}\right)\end{array}$ & $\begin{array}{c}\text { Payback } \\
\text { descontado } \\
\left(\mathrm{R} \$ 10^{3}\right)\end{array}$ \\
\hline \hline 0 & - & & $-296,33^{*}$ & \\
\hline 1 & - & 111,45 & 111,45 & $-184,87$ \\
\hline 2 & - & 105,48 & 105,48 & $-79,39$ \\
\hline 3 & 1 & 8,32 & 8,32 & $-71,07$ \\
\hline 3 & 2 & 8,32 & 8,32 & $-62,75$ \\
\hline 3 & 3 & 8,32 & 8,32 & $-54,43$ \\
\hline 3 & 4 & 8,32 & 8,32 & $-46,11$ \\
\hline 3 & 5 & 8,32 & 8,32 & $-37,79$ \\
\hline 3 & 6 & 8,32 & 8,32 & $-29,47$ \\
\hline 3 & 7 & 8,32 & 8,32 & $-21,15$ \\
\hline 3 & 8 & 8,32 & 8,32 & $-12,83$ \\
\hline 3 & 9 & 8,32 & 8,32 & $-4,51$ \\
\hline 3 & 10 & 8,32 & 8,32 & 3,81 \\
\hline
\end{tabular}

* Investimento

\section{CONCLUSÕES}

Um método de dimensionamento ótimo de gerador fotovoltaico para a minimização dos custos anuais totais com energia elétrica foi apresentado. Para tanto, dados reais de irradiância solar, temperatura ambiente e demanda foram usados mediante simulação de Monte Carlo. O algoritmo de Busca Cuco foi utilizado por se tratar de um método que necessita de poucos ajustes e que se adapta a uma grande quantidade de problemas de otimização.

A instalação do sistema FV se mostrou viável economicamente, uma vez que o LCOE foi bem abaixo do custo da energia convencional e o tempo de retorno do investimento se mostrou bastante atraente, pois é menor do que 3 anos. 


\section{AGRADECIMENTOS}

Mariana R. B. Alencar agradece à CAPES pelo apoio financeiro mediante concessão de bolsa de estudos, sem a qual seria difícil a elaboração deste trabalho.

\section{REFERÊNCIAS}

Atwa, Y. M.; El-Saadany, E.F; Salama, M. M. A. and Seethapathy, R (2010). Optimal Renewable Resources Mix for Distribution System Energy Loss Minimization. IEEE Transactions on Power Systems, volume (25), No. 1, 360-370.

Chow, T. T.; Hand, J. W.; \& Strachan, P. A (2003). Buildingintegrated photovoltaic and thermal applications in a subtropical hotel building. Applied Thermal Engineering, 23, volume (16), 2035-2049.

Dalton, G. J.; Lockington, D. A.; Baldock, T. E (2009). Feasibility analysis of renewable energy supply options for a grid-connected large hotel. Renewable Energy, volume (34), 955-964.

IBGE. Intituto Brasileiro de Geografia e Estatística (2016). Pesquisa de Serviços de Hospedagem 2016. Disponível em:

https://ww2.ibge.gov.br/home/estatistica/economia/come rcioeservico/psh/2016/default.shtm. Acesso em: 02 mar. 2019.

INSTITUTO IDEAL E AHK-RJ (2018). O Mercado Brasileiro de Geração Distribuída Fotovoltaica - Edição 2018. Relatório técnico. Disponível em: http://ahkbusiness.de/fileadmin/ahk_business_br/05_Pub licacoes-

Publikationen/O_Mercado_Brasileiro_de_Geracao_Distr ibuida_FV_-_2018.pdf. Acesso em: 01 abr. 2019.

Liu, Z.; Wen, F.; Ledwich, G (2011). Optimal siting and sizing of distributed generators in distribution system considering uncertainties. IEEE Transaction on Power Delivery, No. 4, volume (26), 2541-51.

Nadjemi, O.; Nacer, T.; Hamidat, A.; Salhi, H (2017). Optimal hybrid PV/wind energy system sizing: application of cuckoo search algorithm for Algerian dairy farm. Renewable and Sustainable Energy Reviews, volume (70), 1352-65.

Nishimoto, E.; Varajão, G. F. D. C (2018). Energia solar fotovoltaica em meios de hospedagem no Brasil: nicho de mercado ou tendência à massificação? Cultur, ano 12, volume 1, 148-168.

Oliveira, L. W.; Maria, T. C. J (2017). Planning of renewable generation in distribution system considering daily operating periods. IEEE Latin America Transactions, No. 5, volume (15), 901-907.

Oluseyi, P. O.; Babatunde, O. M. e Babatunde, O. A (2016). Assessment of energy consumption and carbon footprint from the hotel sector within Lagos, Nigeria. Energy and Buildings, volume (118), 149-161.

Pereira, M. C.; Joyce, A.; Reis, P. C (2016). O valor e o custo da electricidade produzida por sistemas solares (fotovoltaicos). Relatório técnico. Disponível em: http://www.ipes.pt/ipes/wpcontent/uploads/2017/10/IPES-O-valor-e-o-custo-daelectricidade.pdf. Acesso em: 01 mar. 2019.
Qi, Y.; Jianhua Z.; Zifa L.; Shu X.; Weiguo L (2008). A new methodology for optimizing the size of hybrid PV/wind system. IEEE ICSET; 922-7.

Veríssimo, P. H. A (2017). Avaliação de uma usina solar fotovoltaica comparando diferentes topologias de sistemas de fixação utilizando duas diferentes tecnologias FV. Dissertação de mestrado. Universidade Federal de Santa Catarina.

Shan, S (2019). Power consumption data of a hotel building, IEEE Dataport. [Online]. Disponível em: http://dx.doi.org/10.21227/29hy-9380. Acesso em: 22 mar. 2019.

Stoppato, A.; Benato, A.; Destro, N. e Mirandola, A (2016). A model for the optimal design and management of a cogeneration system with energy storage. Energy and Buildings, volume (124), 241-247.

Yang, X-S. e Deb. S (2009). Cuckoo search via Lévy flights. Proceeding of the World Congress on Nature \& Biologically Inspired Computing (NaBIC 2009). 